\title{
Disparate Electrospray Systems for Undergraduate and Graduate Education
}

\section{Dr. Amelia Greig, California Polytechnic State University, San Luis Obispo}

Dr Amelia Greig has degrees in Mechanical and Aerospace Engineering, and Science from the University of Adelaide, and a PhD in Physics from the Australian National University. She teaches courses in spacecraft propulsion and the space environment at Cal Poly San Luis Obispo, and also leads the Aerospace Engineering Department's micro-propulsion research activities.

\section{Mr. Alex Powaser, California Polytechnic State University, San Luis Obispo}

Alex is a graduate student in Aerospace Engineering at California Polytechnic State Universtiy, San Luis Obispo. His thesis work is on developing a colloid thruster to be used at Cal Poly's undergraduate laboratory. While being a student, Alex enjoys teaching various labs and lectures for the university as well. Outside of the classroom, he enjoys an active leadership role in CRU (formally known as Campus Crusade for Christ) and other hobbies such as playing guitar, being an amateur woodworker, and exploring California's Central Coast with his fiancée.

\section{Douglas Howe, California Polytechnic State University, San Luis Obispo Mr. Will Alan McGehee, California Polytechnic State University}

Will McGehee is currently attending California Polytechnic State University (Cal Poly) in pursuit of his Master's Degree in Aerospace Engineering. He completed his undergraduate degree in Aerospace Engineering at Cal Poly as well. His thesis encompasses the design and initial operational testing of an electrospray (aka colloid) thruster for current and future research efforts at Cal Poly in the field of micropropulsion. He has experience in electrical systems, micro-fabrication, and diagnostic techniques used in testing of micro-propulsion thrusters. Additionally, he has experience in the design, fabrication, and assembly of systems including electrical, composite, and traditional material components. 


\title{
Disparate Electrospray Systems for Undergraduate and Graduate Education
}

\begin{abstract}
Electrospray thrusters are low thrust, high efficiency devices that use electrostatic fields to accelerate droplets of non-volatile liquid propellants. With numerous applications in precision attitude control and propulsion of small satellite platforms, electrospray systems are gaining prevalence in the field of electrostatic propulsion. These thrusters present unique educational opportunities to expose students to comparatively novel technologies in a laboratory setting, requiring only modest university resources and development time.
\end{abstract}

In support of its "Learn by Doing" pedagogical philosophy, the Aerospace Engineering Department at California Polytechnic State University in San Luis Obispo, CA has developed electrospray thruster platforms for both graduate research and education and course-based undergraduate education purposes. The research-oriented platform has fidelity to practical applications, ensuring modularity to support a wide variety of potential research studies. In contrast, the undergraduate lab electrospray thruster is inexpensive to construct and maintain, focuses on demonstration of core principles, and provides students an interactive experience.

\section{Introduction}

The aerospace industry is continually expanding and developing new technologies. Academic institutes must keep up with these changes to technology and their applications so students enter the workforce as educated engineers and scientists capable of continuing the growth of the industry. As technologies advance, the manufacturing and scientific processes involved can become more difficult, and it can be hard to develop quick, simple demonstrations for student lab classes.

One example of this is in the field of electrostatic propulsion. In electrostatic propulsion, a low mass flow rate of propellant is accelerated to high exhaust velocities using electrostatic fields. Gridded ion thrusters and Hall thrusters are the two most common electrostatic propulsion devices, but these can be difficult to build and operate in standard workshops, particularly when concerning grids for ion thrusters and the ceramic annular channel in Hall thrusters. To teach the principles of electrostatic propulsion, a different device known as an electrospray thruster may be used instead. Electrospray thrusters are significantly simpler to make and, although the operation is somewhat different to gridded ion and Hall thrusters, the basic principles of electrostatic propulsion can be accurately demonstrated with the electrospray system.

Electrospray thrusters are not a new technology, being first developed in the 1960's [1]. Initially they were not popular due to low thrust levels and high voltages required. The emergence of 
micro- and nano- satellite technologies has seen a resurgence in interest in electrospray systems as there are more avenues for their use and improvements in technology have dropped the required voltages [2,3]. Electrospray thrusters are now being considered as high specific impulse (propellant efficient), low thrust devices for small satellite maneuvering and control $[4,5]$.

Electrospray thrusters for flight missions are a complex technology, requiring a balance of electrical performance, fluid flow management in micro-gravity, and manufacturing precision for high performance, and system lifetimes. However, the basic principles of the devices can easily be demonstrated in a lab setting as part of an undergraduate curriculum using a simplified terrestrial version of the thruster to demonstrate key principles and operational considerations. Minor modifications can be made to the such a set-up to convert it to a thruster suited to a graduate level curriculum where the realistic aspects of operating in a space environment can be better represented and studied.

This paper outlines the use of simple, low cost electrospray devices in a spacecraft propulsion curriculum setting for both undergraduate and graduate level. Methodology, challenges, and pedagogical goals will be addressed, finishing with a summary of student experiences in the years this lab has been employed in the curriculum.

\section{Electrospray Thrusters}

Electrospray thrusters use electrostatic fields to emit minute propellant particles, either small droplets or single ions, to produce thrust. The propellant is stored in liquid form, with the emitted particles drawn from the surface of the electrified liquid propellant through Taylor cone or conejet formation. Within the electrospray thruster group there are a number of sub-categories, including colloid thrusters and field emission electric propulsion (FEEP) [6], the former trading total thrust for propellant efficiency by emitting individual ions while the latter increases total thrust by emitting larger droplets.

The main components of an electrospray thruster, shown in Figure 1, are the propellant storage and feed system that maintains a constant surface of propellant at the end of an emitter tip, and a charged extractor grid that produces the electrostatic field for particle extraction and acceleration. In some cases, an acceleration grid is added to enhance thrust and prevent backscattered propellant from damaging the thruster. A neutralizer is also required as the particle beam contains particles of only a single charge which must be charge neutralized before it can detach from the thruster to produce thrust. In some cases, there have been bipolar electrospray arrays developed where multiple emitters alternate from expelling positive and negative charges thereby creating a quasi-neutral beam that requires no further neutralization [7]. However, these devices are significantly more complex and are not considered here where simplicity and ease of manufacture are key aspects. 


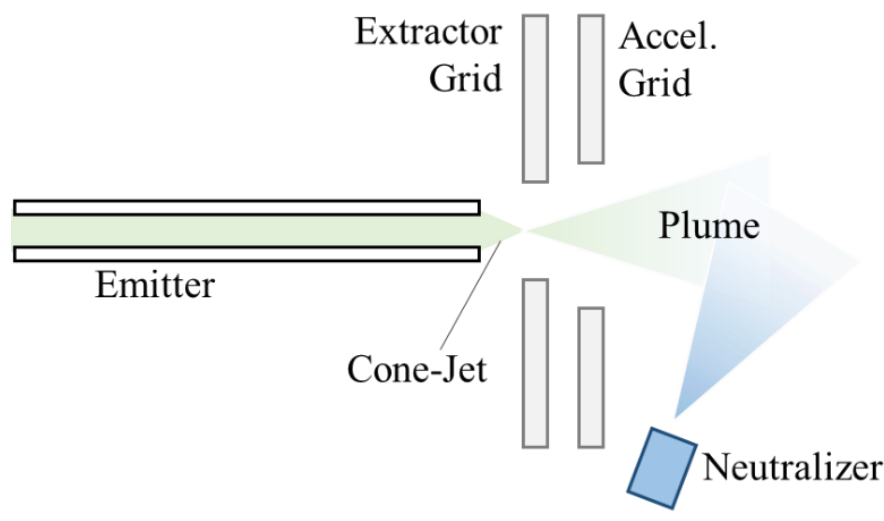

Figure 1: Standard Electrospray Configuration

A potential difference is applied between the emitters and the extractor plate to develop a strong electric field near the emitter tip. The electric field electrifies the propellant near the surface and a Taylor cone forms $[5,6]$. The electric field strength is concentrated near the infinitely small tip of the Taylor cone, and the electrostatic forces overcome surface tension removing particles from the surface and accelerating them away from the thruster. In practice however, the bead that forms at the end of the emitter is not a true Taylor cone as the tip never reaches infinitesimal size, so often the term cone-jet is used instead [8].

There are three types of emitters, the externally wetted, internally wetted, and porous tip emitter, as shown in Figure 2. In each case, the propellant is drawn to the emitter tip by capillary action and surface tension forces.

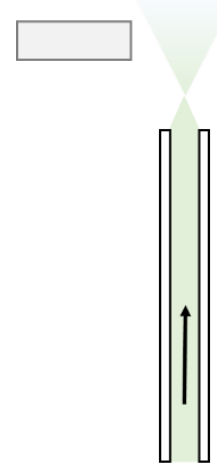

(a)

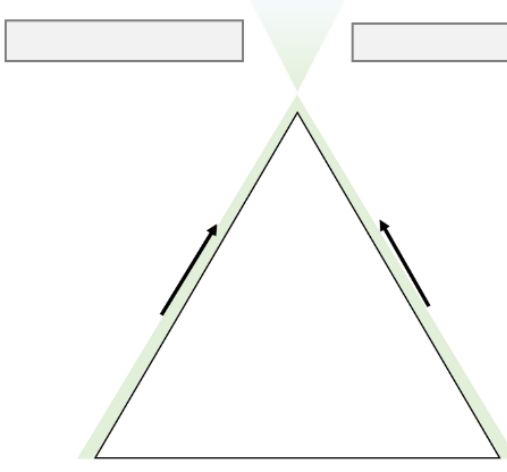

(b)

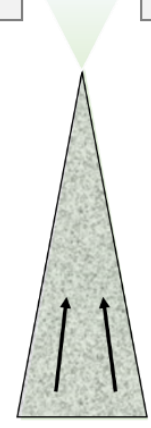

(c)

Figure 2: Electrospray emitter types: (a) internally wetted, (b) externally wetted, and (c) porous tip

For commercially developed thrusters or those specifically designed for flight purposes, a large number of emitter tips in as small an area as possible is desirable to maximize thrust density. In these cases, microelectromechanical systems (MEMS) manufacturing techniques are being employed in general, with emitter tip densities up to $1600 / \mathrm{cm}^{2}$ [9]. However, it is possible to 
construct a fully functional electrospray thruster using much larger emitters that do not require MEMS manufacturing. One of the simpler methods is to use standard blunt tip syringe needles as internally wetted emitters.

The emitted particles accelerate through small holes in the extractor grid and away from the thruster. The thrust produced $(T)$ is proportional to the speed the propellant droplets (exhaust) leave the thruster $\left(v_{e}\right)$ and the mass flow rate of propellant $(\dot{m})$, as represented in Equation 1 .

$$
T=\dot{m} v_{e}
$$

The velocity with which particles leave the thruster depends on the voltage applied between the extractor plate and the emitters, or between the emitters and accelerator grid if using an acceleration grid. The particles are accelerated by an electrostatic force, reaching the velocity given by Equation 2, where $V_{a}$ is the applied voltage and $\frac{q}{m}$ is the charge to mass ratio of the emitted particle.

$$
v_{e}=\sqrt{2 \frac{q}{m} V_{a}}
$$

While electrospray thrusters can achieve exhaust velocities several orders of magnitude higher than chemical systems, the mass flow rate is very low resulting in low overall thrust. The high exhaust velocity does however give them an increased specific impulse over chemical thrusters. Specific impulse $\left(I_{s p}\right)$, represented by Equation 3 where $g_{0}$ is standard gravitational acceleration, is measure of the amount of thrust that can be generated for a given amount of propellant mass.

$$
I_{s p}=\frac{T}{\dot{m} g_{0}}
$$

Higher specific impulse indicates a decrease in the total amount of propellant required to complete a certain mission. However, because increases in $I_{s p}$ results in decreased thrust for electrosprays it must be considered if lower spacecraft mass or higher thrust is more important for a given mission. Operation in droplet mode uses a higher propellant mass flow rate to achieve higher thrust but still experiences exhaust velocities comparable to the ion emission mode, therefore producing lower $I_{s p}$. The choice of which mode to operate in is a design choice dictated by required mission parameters.

The development and operation of electrospray thruster requires knowledge of electrostatics and fluid mechanics as well as general propulsion fundamentals and those related specifically to electrostatic propulsion. Through studying electrospray design and operation, the knowledge garnered can be transferred to other applications. In particular, the electrostatic acceleration of 
the droplets is transferrable to other forms of ion thrusters, which are more common but harder to implement in an educational lab setting. Additionally, as a somewhat unknown technology, students will approach the topic with minimal preconceptions that may inhibit learning [10].

\section{Undergraduate Lab Electrospray Thruster}

An electrospray thruster designed specifically for use in an undergraduate lab class is outlined in this section. This system is developed to teach principles of electrospray operation, electrostatic propulsion, and general measurement and analysis of propulsion system performance.

\subsection{Design Methodology}

When designing the electrospray thruster for the undergraduate lab class, high performance in regard to thrust or specific impulse is a secondary consideration. Instead, the focus is on the pedagogical aspects.

For maximum learning impact [11], the set-up is designed for tactile and visual interactions with the students. The system accurately represents the key principles of electrospray operation including electric field application, cone-jet formation, capillary action for propellant feed, and acceleration of charged particles for thrust. The system is comparable in design and operation to commercially available electrospray systems, and students can make direct measurements of performance without specialized equipment such as high precision thrust balances [12].

To minimize system costs and ensure material acquisition is not a limiting factor, the system uses readily available, low cost materials and components. A larger system operating in the droplet emission mode eliminates the need for advanced MEMS manufacturing when constructing the emitter array and allows students to observe emitter design and cone-jet formation with the naked eye. Finally, applied voltages are kept as low as possible to minimize potential safety hazards to students.

\subsection{Electrospray Design}

The electrospray thruster designed for use in an undergraduate lab class is shown in Figure 3. The system has a propellant reservoir fed by gravity to the emitter array cavity. Such a propellant feed system would not work in microgravity, but is employed here to maintain system simplicity. The drawing of the propellant through to the emitter tip still occurs primarily through capillary action, and a bead forms at the tip of the emitter that the student can observe. The propellant used is glycerol, as it is easy to obtain, inexpensive, and non-toxic [13]. Glycerol doped with ionic compounds may be used for enhanced performance should it be desired. 

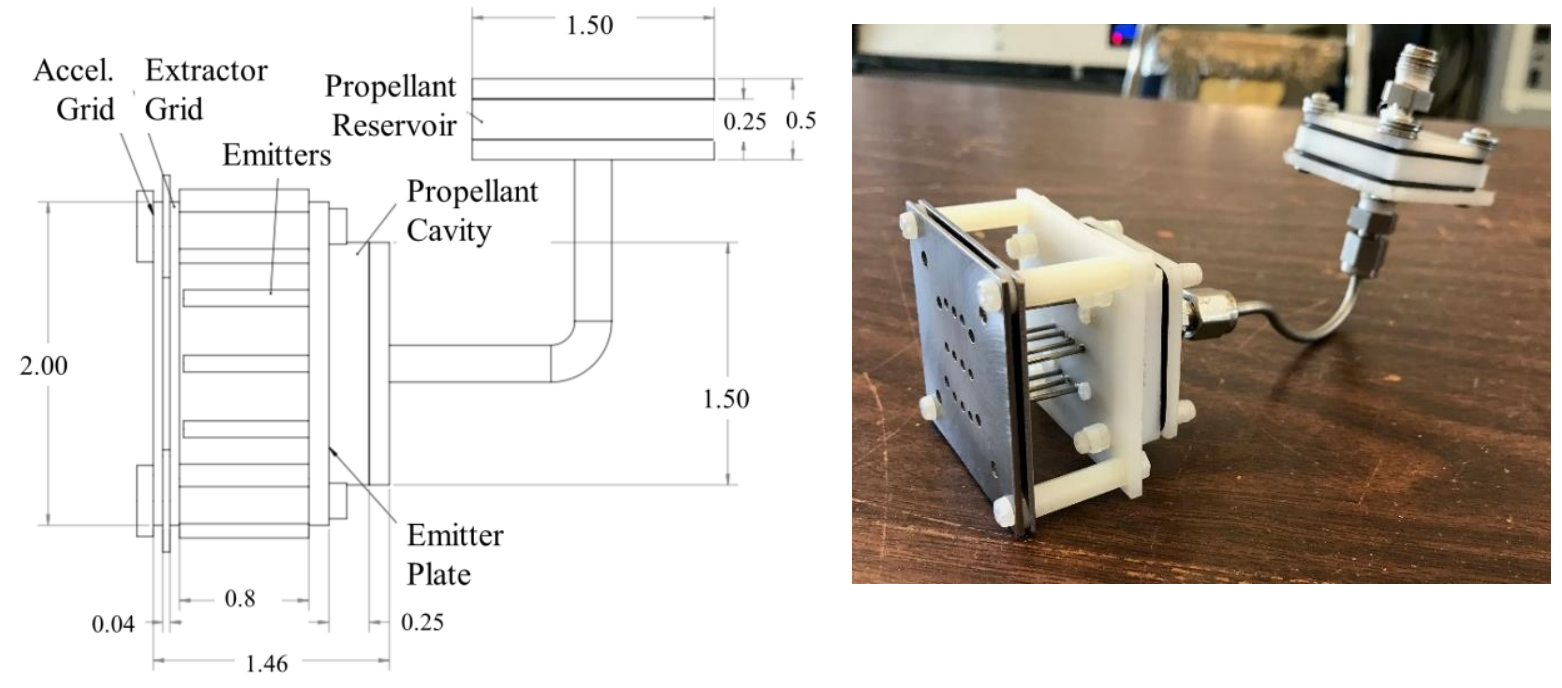

Figure 3: Electrospray thruster for undergraduate lab use

The emitters are 18-gauge blunt tip syringe needles soldered to an emitter plate constructed from stainless steel with corresponding propellant feed holes. Blunt tip syringe needles act as internally wetted emitters, which are typically larger in size, thus increasing visual impact for students as well as easing fabrication and maintenance. An array of 10 emitters is used, arranged in a dimensionally scaled-up fashion corresponding to the emitter spacing used on microscopic arrays [13]. The scaled-up version lets the student see the design more easily and makes for easier manufacturing. A single emitter tip could be used, but a planar array is more realistic for comparison to commercial and flight hardware systems $[1,14,15]$ and produces higher thrust levels for measurements in the lab.

For smaller devices, the cone-jets and emitted droplets cannot be seen with the naked eye. However, for larger devices such as the one described here, these phenomena can be observed with the naked eye, as shown in Figure 4. Figure 4(a) shows the formation of a propellant bead from capillary action and surface tension alone, prior to the application of an electric field. Figure 4(b) shows the elongation of the bead towards cone-jet formation under an applied electric field.

The extractor and accelerator grids are constructed from stainless steel as it is readily obtained and easily machined. The system must be designed such that the extractor surface is as close as possible to the emitters to establish a strong electric field. Electrical isolation of the system is managed with HTPE, a low cost, easily machined insulating material. The vast difference in coloring of the conductive and insulating materials visually displays the importance to electrical isolation of high voltage components. Use of screws and spacers make the thruster easy for the students to disassemble, reassemble, and operate the thruster in a single session. 


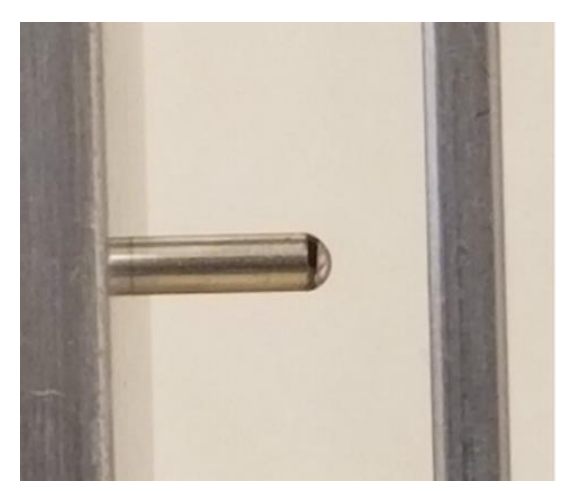

(a)

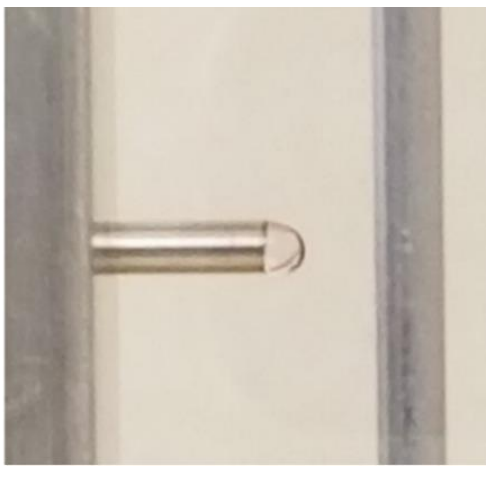

(b)

Figure 4: Single emitter tip showing (a) bead formation from capillary action and (b) elongated bead forming a cone-jet under application of electric field (right) for a single emitter tip.

To eliminate the need for specialized high-voltage supplies, the applied voltage across the system is kept as low as possible. From space charge limited current density [6], the applied voltage $\left(V_{a}\right)$ to produce a defined thrust density $\left(\frac{T}{A}\right)$ for a given emitter spacing $(d)$ is given in Equation 4 , where $\epsilon_{0}$ is the permittivity of free space. The area $(A)$ is the combined liquid surface area of the emitter tips across the array.

$$
V_{a}=\frac{3}{4} d \sqrt{\frac{2 T}{\epsilon_{0} A}}
$$

Applied voltages are on the order of a few hundred Volts. To achieve these values, two Glassman $1 \mathrm{kV}$ power supplies are used. One power supply is connected to the accelerator grid, while the other is used to establish the potential difference between the extractor grid and the emitters. A schematic of the electric set-up of the thruster is shown in Figure 5.

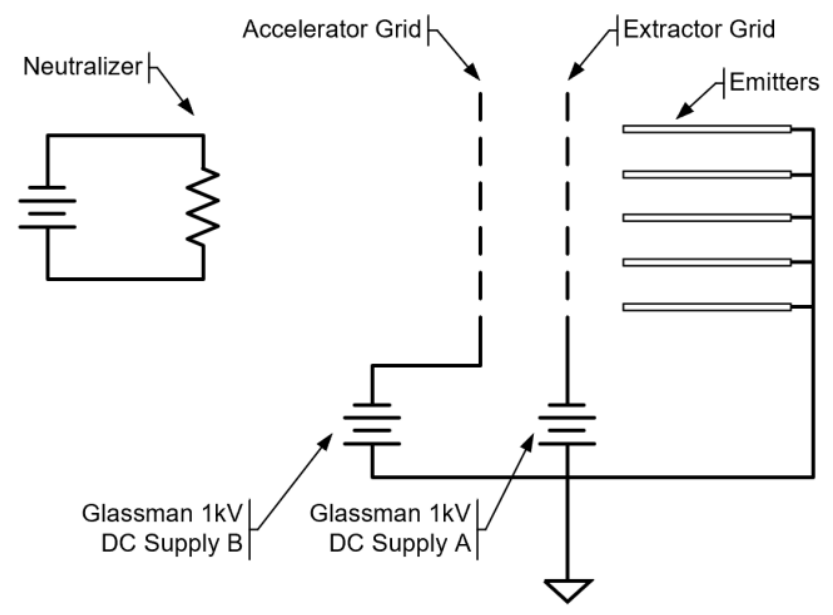

Figure 5: Electrical schematic of the electrospray thruster system. 
Power $(P)$ required for the system is given by Ohm's Law (Equation 5), where $I$ is the current through the system.

$$
P=I V_{a}
$$

Currents in electrospray systems are very low, on the order of nanoAmps [6], requiring picoammeters for direct measurements. However, if a picoammeter is not available, the current can be estimated using the following method [3]. The current through the system is equivalent to the current carried by the emitted propellant, represented by Equation 6, where $\frac{q}{m}$ is the charge to mass ratio, $\dot{m}$ is the propellant mass flow rate, and $\rho$ is the propellant mass density.

$$
I=\frac{q}{m} \dot{m}=\frac{q}{m} \cdot \rho Q
$$

The volumetric flow rate of the propellant $(\mathrm{Q})$ can be estimated with equation 7 [3], where $\gamma$ is the surface tension of the propellant, $\sigma$ is the electrical conductivity of the propellant, and $\epsilon$ is the relative permittivity of the propellant. The function $f(\epsilon)$ accounts for effects of internal fields and conduction dependencies $[3,13]$.

$$
Q=\frac{\gamma \sigma}{\epsilon\left(\frac{q}{m} \frac{\rho}{f(\epsilon)}\right)^{2}}
$$

In practice, the propellant mass flow rate can also be estimated by weighing the total propellant used through a test and dividing the mass by the thruster operational time.

As propellant is extracted from the emitters, some of it may hit the grids rather than passing through to contribute to thrust, thus reducing the propulsive efficiency of the system. For a more realistic estimate of thrust, the propellant mass flow rate used in the thrust equation (Equation 1) should include only the mass that passes through the extractor and accelerator grids. A downstream collector plate, oppositely biased relative to the emitted particles, attracts and collects the propellant. The mass of propellant on the plate after a test indicates the mass of propellant successfully passing through the grids. Dividing this by total test time gives average propellant mass flow rate for thrust generation.

Although the collection plate acts somewhat like a neutralizer, a simple filament neutralizer is added to the set-up to instruct the students on plume neutralization and plume detachment [16]. A 0.005" diameter tungsten wire carrying a current of a few Amperes to initiate thermoelectric emission is attached downstream of the accelerator grid as a visual representation of a neutralizer, as seen in Figure 6. 


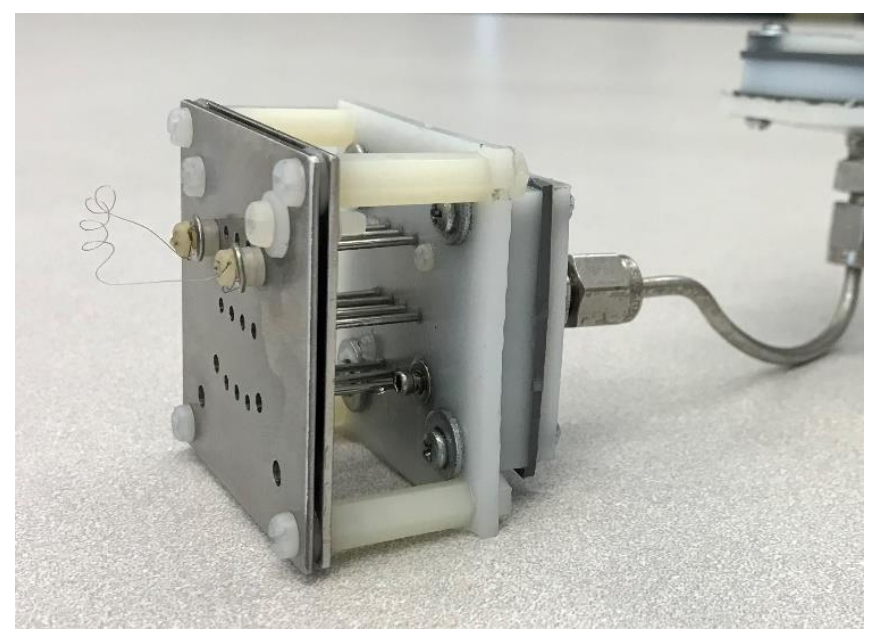

Figure 6: Electrospray thruster for undergraduate lab use showing demonstration filament neutralizer downstream of the accelerator grid.

Combining the propellant flow rate with applied voltage to give an estimate of propellant exit velocity with Equation 2, provides an estimate of thrust produced using Equation 1. Specific impulse is determined through Equation 3, and total power required is determined through Equations 5, 6, and 7. Through these calculations, students analyze performance of the thruster under different conditions and draw conclusions based on the theory outlined. Example test data is presented in Table 1.

Table 1: Example thruster measurements and performance estimates.

\begin{tabular}{c|c|c|c|c|c}
$\begin{array}{c}\text { Applied } \\
\text { Voltage }(\mathrm{V})\end{array}$ & $\begin{array}{c}\text { Collected } \\
\text { Mass }(\mathrm{mg})\end{array}$ & $\begin{array}{c}\text { Test Duration } \\
(\mathrm{min})\end{array}$ & $\begin{array}{c}\text { Mass Flow } \\
\text { Rate }(\mathrm{mg} / \mathrm{s})\end{array}$ & $\begin{array}{c}\text { Thrust } \\
(\mu \mathrm{N})\end{array}$ & $\begin{array}{c}\text { Specific } \\
\text { Impulse }(\mathrm{s})\end{array}$ \\
\hline 1000 & 7.5 & 5 & .025 & 311.2 & 1270 \\
\hline 900 & 5.7 & 5 & .019 & 246.8 & 1325 \\
\hline 800 & 2.5 & 5 & .008 & 129.5 & 1585 \\
\hline 700 & 1.8 & 5 & .006 & 96.3 & 1684
\end{tabular}

Student assessment for the lab was structured as part calculation and part general knowledge of electrospray thruster applications to spacecraft propulsion. Performance estimates, such as those given in Table 1, were made with the students then applying the results to particular satellite missions suited to electrospray applications. Based on the high specific impulse and low thrust values calculated, example mission options could include precision attitude control, orbit maintenance, or small satellite formation maneuvering.

\subsection{Atmospheric operation}

Operating the thruster in a vacuum chamber is more realistic in terms of spacecraft operations and additionally provides a safety barrier between students and the high voltage components. However, if a vacuum chamber is not available, thrusters may be operated under atmospheric 
conditions. One atmosphere of pressure pushing back on the emitter tip is too strong for capillary action alone to function as a propellant feed mechanism. A syringe pump is instead used to control to propellant flow rate through and help form the propellant beads required for cone-jet formation. The syringe pump may further serve as an analog to flow control systems used in flight applications such as piezovalves. The set-up of the atmospheric device is shown in Figure 7. Here only a single emitter tip is used.

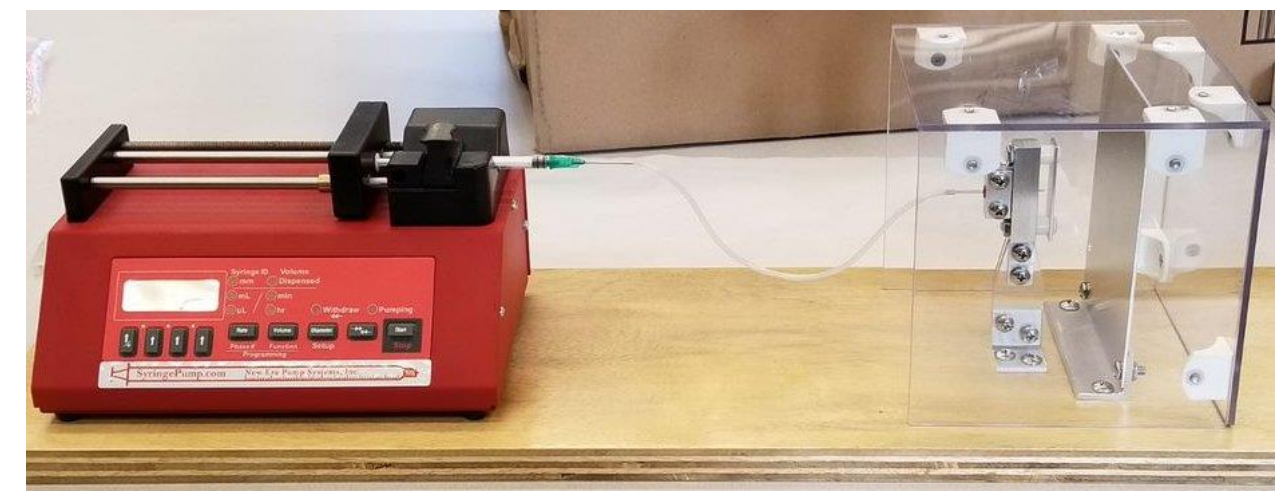

Figure 7: An electrospray thruster with single emitter tip fed with glycerol propellant from a syringe pump for use in atmospheric pressure.

\subsection{Student Experience}

A comparison between students exposed to the lab to students who were not exposed to the lab is made. Student sample 'A' contains 67 students who undertook the spacecraft propulsion course prior to the implementation of the electrospray lab described here. Student sample 'B' contains 72 students who undertook the spacecraft propulsion course including the electrospray lab. The addition of the electrospray lab to the curriculum is the only significant change to course structure between the two samples.

Both sample groups were asked questions directly related to electric propulsion performance and sizing. One question required them to appropriately size an electrospray thruster system for a small satellite mission to an inner planet. Another question related to calculating the performance of other forms of electric propulsion systems not directly included in the lab (e.g. gridded ion thruster and resistojet). Further questions related to integration of thrusters into spacecraft including considerations such as power.

The average score of sample A (no electrospray lab) is $85.2 \%$ with a standard deviation of $6.0 \%$. The average score of sample B students (with electrospray lab) is $84.9 \%$ with a standard deviation of $5.7 \%$. These results show no statistical difference between scores for students who participated in the electrospray propulsion lab compared to those who did the course without the electrospray propulsion course. However, the sample sizes are limited to two classes of students only and further analysis of student scores will continued to be performed as more students are 
exposed to the electrospray lab, increasing the sample size and statistical significance of the results.

While the implementation of this lab is still in its infancy, limiting the significance of any statistical analysis to gauge student learning, students generally expressed enjoyment and positive comments about the lab experience. Students expressed that using a macro-scale thruster with which they could interact and which they could observe directly was enjoyable and helpful for learning. Others noted that the use of the thruster helped to distinguish between thruster types, with one student remarking "It was very interesting to get to use a real thruster. I didn't have a great understanding of the differences between different types of electric propulsion, and it was very interesting to get to see a real-world example of one type."

\section{Graduate Education Electrospray Thruster}

An electrospray thruster system designed for use in a graduate level curriculum is outlined throughout this section. The thruster is designed to more realistically replicate commercially available technologies and flight qualified electrospray systems for the students to contribute meaningful research to the field. However, the cost and complexity of the system remains low. Schools with highly developed electrospray programs may have access to higher levels of funding to enable MEMS manufacturing of components and specialized equipment for advanced instrumentation. The thruster outlined here is designed for a school wishing to establish an electrospray program or to simply introduce students to the more advanced aspects of developing electrostatic propulsion systems and is design to be low cost,requiring only simple materials and components to establish.

As with the undergraduate system previously described, the set-up is designed for tactile and visual interactions with the students to maximize learning impact [11]. Retaining larger emitter sizing, as with the undergraduate system outline above, lets graduate students easily visualize and study key operation principles such as propellant bead formation prior to electric field application.

A schematic of the thruster system is given in Figure 8. The system contains five emitter tips made from 18-gauge blunt stainless steel surgical needles arranged in a linear fashion and an extractor plate with five individual holes. A single Glassman 10kV DC power supply is used to establish a strong electrostatic field (up to $10 \mathrm{kV}$ ) between the emitters and extractor plate. The propellant is fed by a passive capillary action system consisting of five small tubes, one for each emitter. 


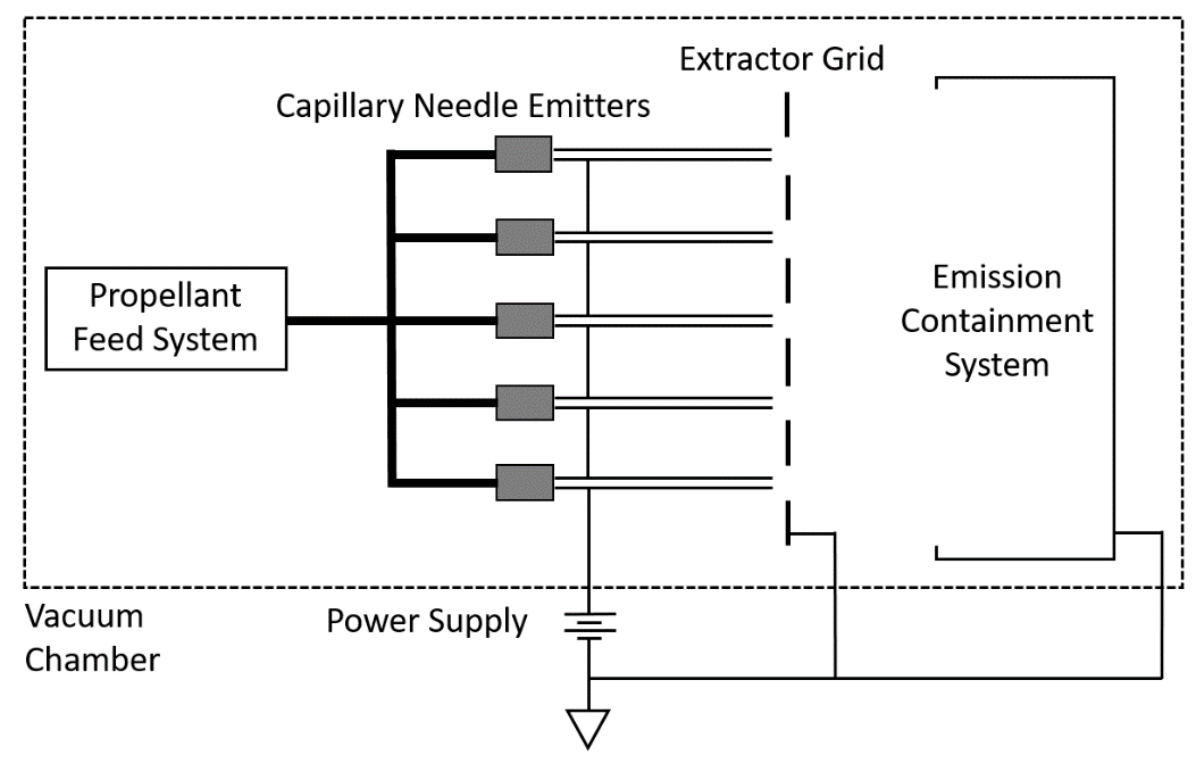

Figure 8: Schematic of a low-cost electrospray thruster designed for graduate education.

A photo of the system in a vacuum chamber is shown in Figure 9(a). The large silver box to the right in Figure 9(a) is the propellant emission containment system used to prevent propellant droplets from entering and contaminating the vacuum pump system. Figure 9(b) is a close-up view of the emitter tips showing the propellant bead formation prior to the establishment of an electric field.

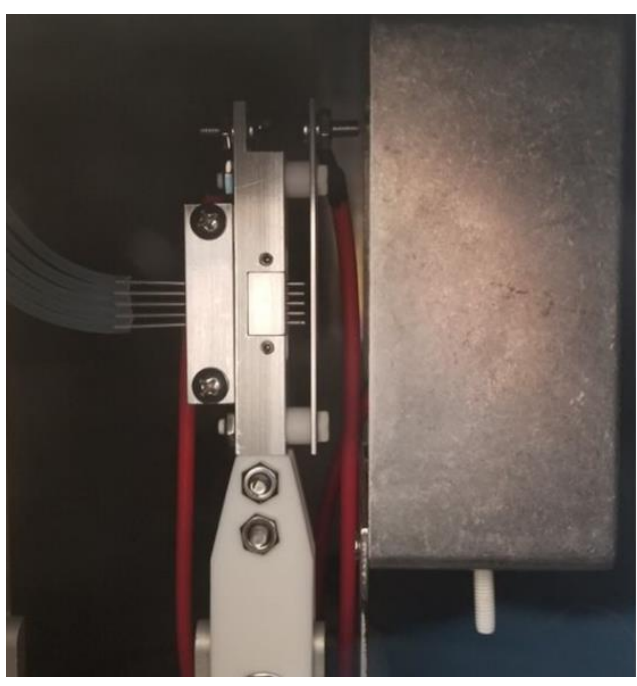

(a)

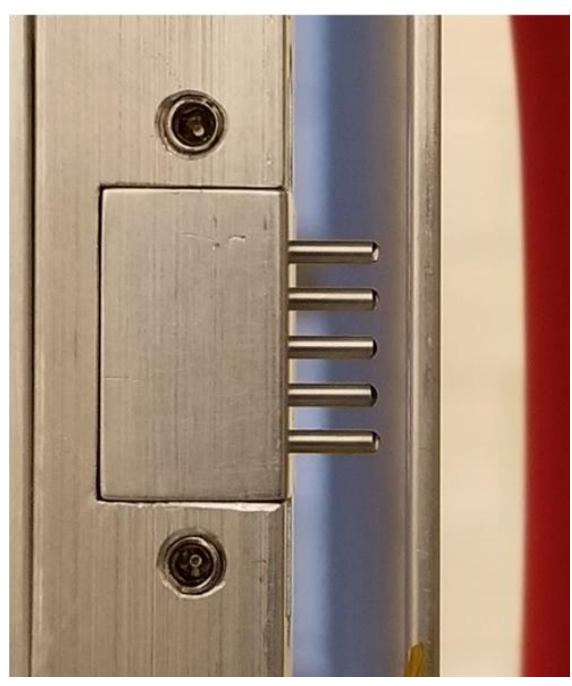

(b)

Figure 9: Electrospray thruster designed for graduate education (a) with magnified emitter tip region showing formation of propellant beads (b). 
Modularity of design is an important factor in the design. Interchangeable emitter array sections (the rectangular block seen in Figure 9(b)) and extractor plates are included so that multiple research projects can be implemented on a single thruster system with minimal modifications. Employing modularity in this manner allows students to perform more experiments in a shorter period, enhancing their skills in designing and implementing experiments. This simple system can perform research into many avenues of investigation, including testing of propellant types, flow rates, propellant feed systems, deposition of propellant on the extractor plate, damage from propellant incident on the extractor plate limiting lifetimes, emitter tip spacing and arrangement, and extractor grid hole placement and sizing. Each of these are currently of great interest to the electrospray development community in attempts to increase performance and lifetime $[1,2,8,14]$.

\section{Conclusions}

Academic institutes training future generations of engineers must continually innovate lab classes to maintain parity with advances in technology. In regards to propulsion, and specifically electrostatic propulsion, electrospray thrusters are an excellent example of simple, low-cost tools for this purpose. Here, two disparate electrospray systems have been developed for use in undergraduate and graduate level curriculum activities. Both systems are low cost, robust, and representative of commercially available or flight-qualified electrospray systems. The system designed for use in an undergraduate curriculum utilizes tactile and visual interactivity to enhance student experiences and can be operated either in vacuum or in atmosphere. The system introduces students to propulsion fundamentals such as thrust and specific impulse as well as more advanced concepts such as electrostatic acceleration of propellant and neutralization. The graduate system utilizes a high degree of modularity to enable multiple experimental research programs to be performed using the same base system.

\section{References}

[1] Lopez Urdiales, J. M. Progress in colloid propulsion, M.Sc thesis, Massachusetts Institute of Technology, Cambridge, MA, 2004

[2] Courtney, D. G., Dandavino, S., and Shea, H., "Comparing Direct and Indirect Thrust Measurements from Passively Fed Ionic Electrospray Thrusters," Journal of Propulsion and Power, Vol. 32, No. 2, March-April 2017. doi: 10.2514/1.B35836

[3] Timilsina, N. "Electrospray Thrusters for Attitude Control of a 1-U CubeSat," MS Thesis, Mechanical and Aerospace Engineering Dept., University of California, Irvine, Irvine, CA, 2014.

[4] Mier-Hicks, F., and Lozano, P. C., "Electrospray Thrusters as Precise Attitude Control Actuators for Small Satellites." Journal of Guidance, Control, and Dynamic, Vol. 40, No. 3, March 2017. doi: 10.2514/1.G000736 
[5] Ziemer, J. K., et al., “Colloid Micro-Newton Thrusters for the Space Technology 7 Mission,” IEEE Paper 978-1-4244-3888-4/10, 2010.

[6] "Session 20: Electrospray Propulsion, MIT OpenCourseWare [online database], URL:

https://ocw.mit.edu/courses/aeronautics-and-astronautics/16-522-space-propulsion-spring-2015/lecturenotes/MIT16_522S15_Lecture20.pdf [retrieved September 2017].

[7] Mahoney, F., Moore, R.D., Perel, J., and Yahiku, Y., "Research and development of a chargedparticle bipolar thruster." AIAA Journal, Vol. 7, No. 3 (1969), pp. 507-511.

https://doi.org/10.2514/3.5137

[8] Gamero-Castaño, M., and Hruby, V., "Characterization of a Colloid Thruster Performing in the microNewton Thrust Range,” International Electric Propulsion Conference, IEPC-01-282, Pasadena, CA, 2001.

[9] Velasquez-Garcia, L. F., Akinwande A. I. and Martinez-Sanchez, M., "A Planar Array of MicroFabricated Electrospray Emitters for Thruster Applications," in Journal of Microelectromechanical Systems, vol. 15, no. 5, pp. 1272-1280, Oct. 2006. doi: 10.1109/JMEMS.2006.879710

[10] Kind, V., Pedagogical content knowledge in science education: perspectives and potential for progress, Studies in Science Education, 45:2, 169-204, 2009, doi: 10.1080/03057260903142285

[11] Schank, R. C., "What We Learn When We Learn by Doing," Cogprints, University of Southampton, Paper ID 637, 1998.

[12] Hicks, F.M., Perna, L., Coffman, C., and Lozano, P.C., "Characterization of a CubeSat compatible magnetically levitated thrust balance for electrospray propulsion systems", 49th AIAA/ASME/SAE/ASEE Joint Propulsion Conference, Joint Propulsion Conferences, (AIAA 2013-3879) https://doi.org/10.2514/6.2013-3879

[13] Physical Properties of Glycerine and Its Solutions, Glycerine Producers' Association, New York, 1963.

[14] Velasquez-Garcia, L. F., "The Design, Fabrication and Testing of Micro-fabricated Linear and Planar Colloid Thruster Arrays," PhD Dissertation, Massachusetts Institute of Technology, Cambridge, MA, 2004.

[15] Hruby, V., Gamero-Castaño, M., Falkos, P., and Shenoy, S., "Micro Newton Colloid Thruster Development," International Electric Propulsion Conference, IEPC-01-281, Pasadena, CA, 2001.

[16] Goebel, D., and Katz, I., "Fundamentals of Electric Propulsion” Wiley, Hoboken, N.J., 2008. 